\title{
Subcutaneous tissue flaps for hallux covering
}

\author{
Luca Vaienti • Victor Urzola • Andrea Scotti • \\ L. Masetto
}

Received: 16 November 2009/ Accepted: 10 December 2009/Published online: 5 February 2010

(C) Springer-Verlag 2010

\begin{abstract}
With the understanding of the extensive vascular supply of the subcutaneous tissue, of its efficacy in the protection of the anatomical structures and of its capability of promoting the adequate functioning of very stressed regions of the human body, the use of subcutaneous adipose flaps has become a valid and sometimes the only reasonable therapeutic weapon in the treatment of small and medium-sized tissue loss. Such a defect represents a common complication of great toe injuries and surgery. Here subcutaneous flap reconstruction is proposed for the treatment of dorsal and medial soft tissue losses of the hallux complicated with infection. Two case are reported. To the best of the authors' knowledge, this application has not been reported in this anatomical site so far. The technique might be worth knowing both for orthopedic and plastic surgeons, as it may represent a safe, less invasive solution for most tegumentary problems of the dorso-medial side of the first ray.
\end{abstract}

Keywords Subcutaneous tissue flap · Turn-over flap · Hallux reconstruction - Tegument infection

\section{Introduction}

Traumatic tissue loss of the foot represents a common problem for the plastic surgeon, and new techniques

L. Vaienti $(\bowtie) \cdot$ V. Urzola $\cdot$ A. Scotti $\cdot$ L. Masetto

U.O. Chirurgia Plastica e Ricostruttiva,

Università degli Studi di Milano,

Ospedale Policlinico San Donato,

Via Morandi 30, 20097 San Donato Milanese, Italy

e-mail: luca.vaienti@unimi.it continue to emerge to improve the treatment. Complex injuries with severe tissue loss can be successfully repaired by using local and regional flaps and microsurgery. Good results can be obtained with fasciocutaneous or muscular free flaps [1], even if the injury to be treated has considerable dimensions and is complicated with bone exposure. Local pedicle or island flaps can still be a valid and useful alternative because of the simple surgical technique and the good esthetic results, particularly at the donor site [2-6].

In the last few decades, knowledge of the vascular anatomy has increased, and new flaps and surgical methods for the management of small to medium-sized skin defects have been developed. The use of subcutaneous tissue flaps and adipofascial turn-over flaps has become a first-rate treatment for these conditions [7].

Subcutaneous flaps started being used after the subcutaneous plexus was studied. This vascular network is located and uniformly represented in all bodily regions, particularly where subcutaneous tissue is more exuberant, as in the abdominal region.

The anatomical studies of cutaneous vascularization carried out by Pearl and Johnson in 1983 represent a contribution of fundamental importance in this field. They demonstrated, first in animals and then in humans, that there is an extensive subcutaneous vascular network between the dense and loose adipose tissue composed of superficial vessels that form a subdermal plexus and of deeper ones that form a subcutaneous system. These two systems are connected by an extensive network of vertical vessels with the vascularized plane located in the midportion of the subcutaneous tissue, separating dense from loose fat $[8,9]$.

In 1986, Gumener gave a precise description of a very well developed vascular subcutaneous plexus in the leg, oriented in the longitudinal direction with many transverse 
anastomoses supplied by septocutaneous and muscular perforator arteries [8, 9].

The vast blood supply and versatility of this tissue make it a valid and safe option. The most important advantage of this kind of flap, composed of subcutaneous tissue only, is that it has excellent plasticity and versatility associated with very low morbidity of the donor region [9]. These flaps can be raised anywhere, independently from the local vascularization. As mentioned before, the subcutaneous plexus is located everywhere, mainly where subcutaneous tissue is abundant. Furthermore, the subcutaneous histological composition provides excellent gliding tissue [8].

According to reports by Lai and Lin in 1992, the most reliable way to predict the survival of a subcutaneous flap is by the ratio of the area of the flap to the area of its base through which the vessels penetrate to supply the subcutaneous plexus. The proportion between areas should ideally be $4: 1$ compared to the traditional method with a 2:1 ratio of width to length described by Milton in 1970 in conventional flaps [10]. The flap is generally left 30-40\% larger than the defect, and, if the base is wide enough, it can be raised without the underlying fascia, which does not enhance the vascular support of the flap, but gives it protection and stability [10].

The flap dimension must be defined by considering the entity of tissue loss and the capability of the subcutaneous tissue to fulfill large tissue losses thanks to the plasticity that the lack of tegument allows and by calculating the area that is unused when turning over the flap [10].

After analyzing these factors, a preoperatory design should be made. Then, the dissection of the flap can begin. The skin is interrupted and the immediate plane dissected to expose the underlying tissue until the exposure of the superficial fascia is achieved; this surgical stage must be carried out carefully with the aim of preserving the vascularization of the dermoepidermal flaps, as well as the integrity of the subcutaneous tissue.

Once the dermoepidermal flaps are raised, the limits of the subcutaneous flap are incised, and the dissection is carried out in a distal to proximal fashion with relation to the area of the lesion and the absolute respect of its base. When the dissection is achieved, the flap is flipped on itself at $180^{\circ}$, the donor area is primarily sutured, and a medium thickness skin graft is apposed over the flap to guarantee its coverage.

Surgical reconstruction of loss of soft tissue of the great toe has always proven to be a difficult problem. This anatomical structure is known to be an essential part of the biomechanical function of the foot, and its functional alteration or absence has proved to lead to new ulcerations and further complications [8, 9].

As simple as it may seem, for different reasons the reconstruction of these particular lesions can sometimes be a complicated labor. Skin grafting cannot be used when deep structures are exposed or damaged [3,4]. The limited vascular structures and the frequent anatomical variations of the dorsal arterial network may render the blood supply scarce, above all in smokers and diabetic patients where vascularization is further compromised. Moreover, this eventuality happens when the anatomical position is inadequate because the donor sites are limited or contraindications are found $[2,3,5]$.

In this report the authors present a successful reconstruction using subcutaneous adipose flaps in difficult cases of small and medium-sized hallux tissue loss complicated with local infection.

The patients provided their consent to the publication of their clinical cases.

\section{Case reports}

\section{Case 1}

A 7-year-old patient who sustained a crush lesion complicated by infection with Staphylococcus aureus developed an infective granuloma of the dorsal aspect of the hallux that jeopardized the ungueal matrix and a portion of second phalanx bone (Fig. 1a). First, we completely excised the granuloma (Fig. 1b). Then, we carried out the dissection of a dermo-epidermal flap (Fig. 1c).

Afterwards the subcutaneous flap was placed upside down over the loss of tissue (Fig. 1d, e). Finally, the flap was covered with a mid-thickness skin graft, to ultimate the hallux reconstruction, and the dermoepidermal pocket was sutured on its original site (Fig. 1f). Three months after surgery, a good result was achieved (Fig. 1g, h).

\section{Case 2}

A 52-year-old patient suffered from septic bursitis of the first metatarso-phalangeal joint of the right foot complicated with exposure of the joint structures (Fig. 2a). A subcutaneous tissue flap was designed, raised and tipped over from the dorsum of the foot to cover the affected area (Fig. 2b, c). The donor site was covered with a local flap (Fig. 2d), and the subcutaneous tissue flap was covered with a mid-thickness skin graft (Fig. 2e). Two months after surgery, the affected area was completely covered, and no functional alteration of the articulation was noticed (Fig. 2f, g, h).

\section{Discussion}

Due to the importance of the hallux when walking, the minor or severe soft tissue loss of the dorsal and medial sides of this anatomical structure demands a timely stable and efficacious coverage. As a matter of fact, in order to 

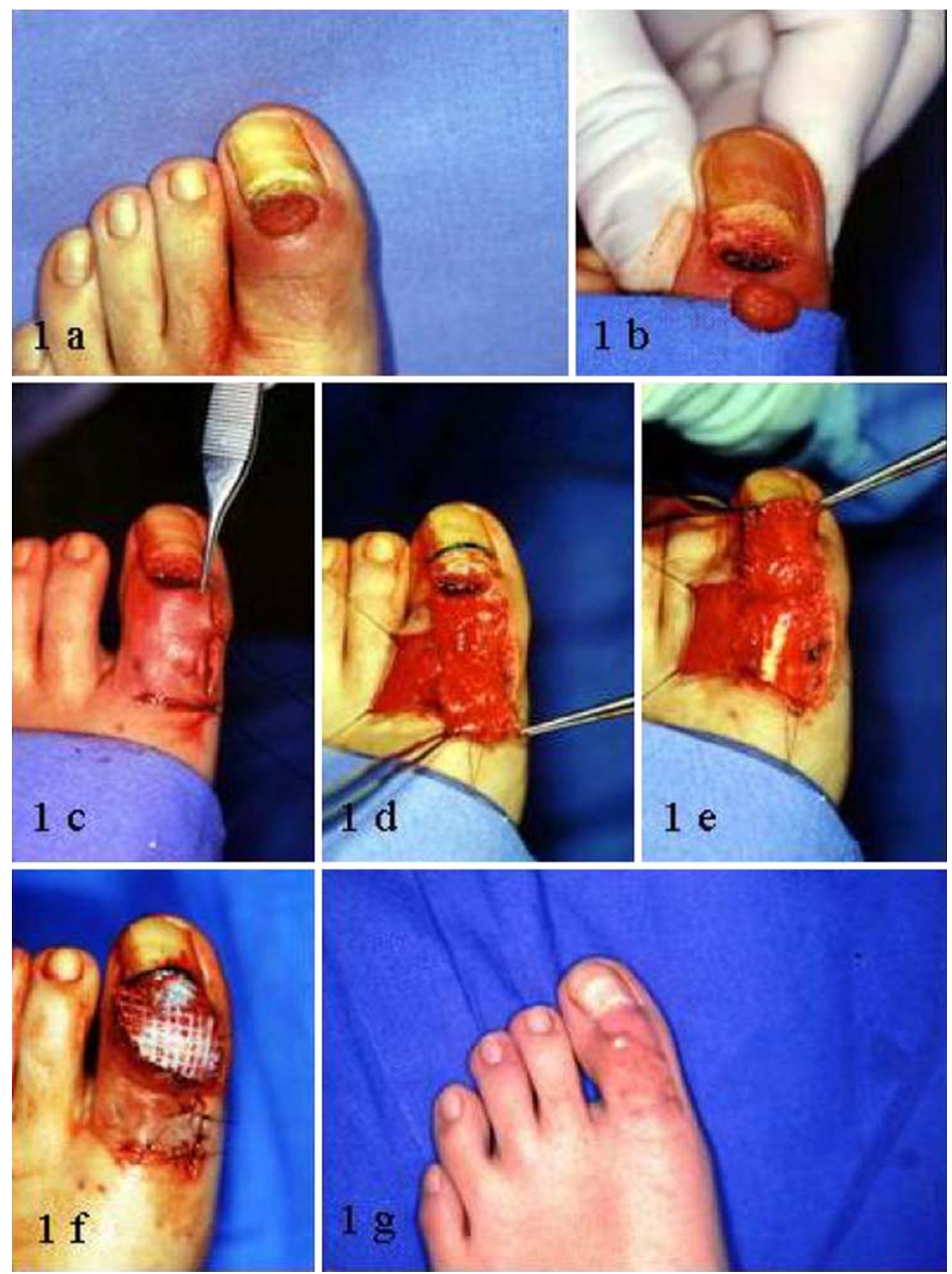

Fig. 1 a Pre-operative view, infective granuloma of the dorsal aspect of the hallux, $\mathbf{b}$ excision of the granuloma, $\mathbf{c}$ dissection of a dermoepidermic flap from the dorsal side of the hallux, $\mathbf{d}$, e the

consider a reconstructive tissue unit suitable for this anatomical site, three fundamental requisites must be present: vitality, capacity to integrate itself with the receiving tissue and the non-production of hypertrophic, dystrophic or painful scars at pressure points on the donor site and on the flap itself.

The last two requirements are fundamental to the reconstruction of the hallux area, which, even though not subcutaneous flap placed upside down over the loss of tissue, $\mathbf{f}$ skin graft and suture, $\mathbf{g}$ follow-up at three months

directly supporting the entire body weight as the plantar region does, suffers, as time goes by, from the inevitable friction caused by wearing shoes. These conditions are aggravated by anatomical deformities, principally those in valgism, of the metatarso-phalangeal articulation and by the mycotic and infective onicodysthrophies.

On the other hand, since they are protected by shoes, the toes are less exposed, in comparison to the fingers, to 

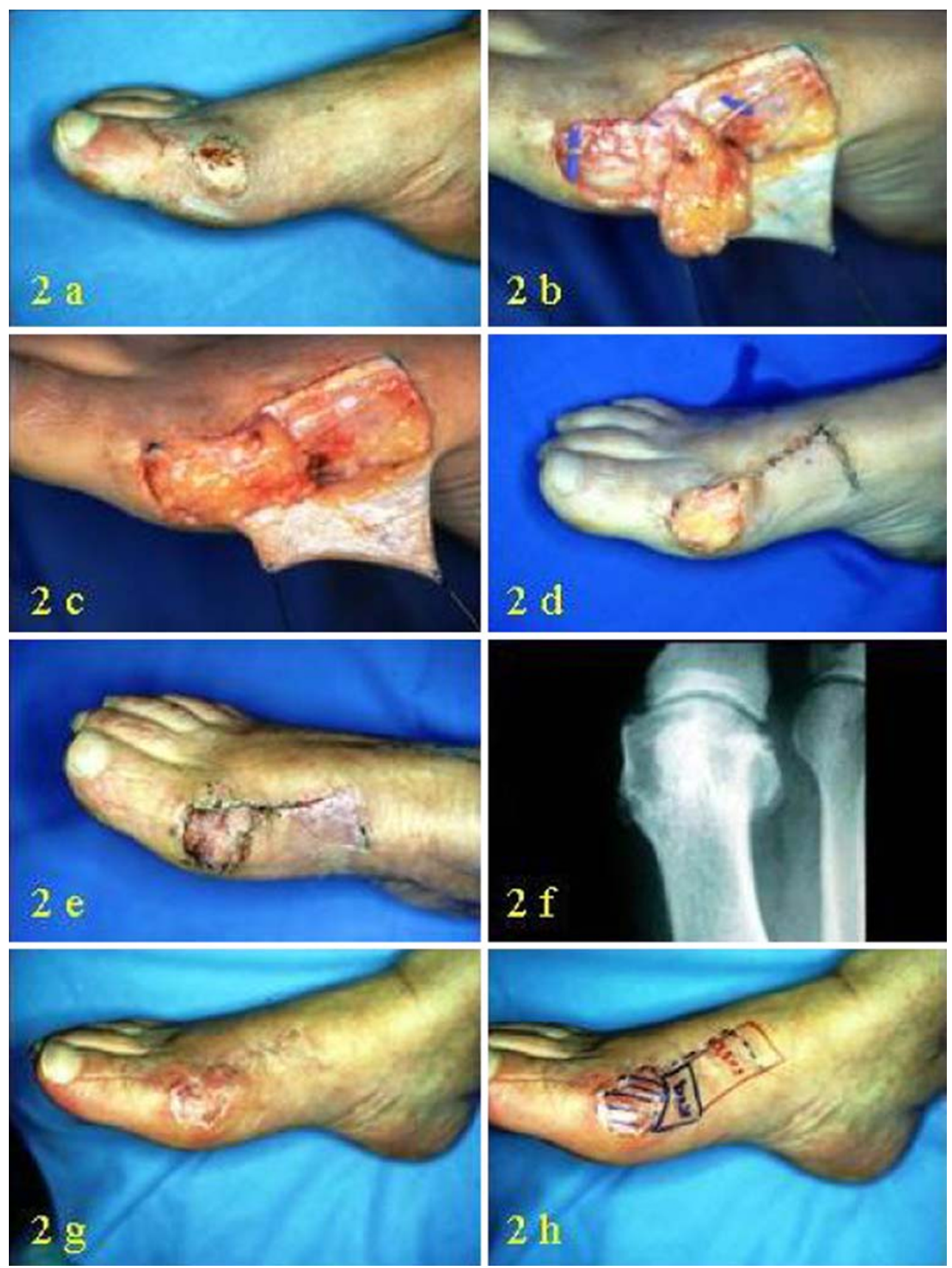

Fig. 2 a Septic bursitis of the first metatarso-phalangeal joint, exposure of the joint structures, $\mathbf{b}$ design and preparation of the flap, respect of the medial nerve of the hallux (blue), c cover of the affected

area, $\mathbf{d}$ a local flap covering the donor side, e mid-thickness skin graft covering the flap, $\mathbf{f}$ X-ray control without signs of infection, $\mathbf{g}, \mathbf{h}$ follow-up at 2 months

traumatic events that produce the true loss of tegument tissue with exposure of underlying structures. At this level, malformative, neoplastic and infective conditions are more frequent.

Therefore, in the presence of a complex lesion appearing on the dorsal or medial surface of the hallux, independent of the etiology of the lesion, an adequate reconstructive procedure from the tegumentary point of view should be

performed because of the primary global importance that the first ray has for the stability and support of the foot.

From a review of the scientific literature relevant to this subject, we found that only a few local, useful homodigital flaps can be considered suitable for covering the dorsalis fascia of the hallux. By considering the evident anatomical and morphological similarities between the hallux and the thumb, and the habitually used flaps for the reconstruction 
of this last structure, the advancement-rotation flap described by Hueston in 1966 is confirmed to be a valid choice for the reconstruction of the loss of tissue of small dimensions. This surgical technique is safe and, when used for the dorsal and medial aspects of the hallux, it does not leave scars in critical supporting areas. The base of the integration graft required for this area has a dimension that usually does not produce significant sequelae. Other viable flaps gained from hand surgery for the reconstruction of the volar aspect of the hallux are the desepithelialized crossfinger flaps. Well known is the role of a cross-finger flap from the second toe in reconstruction of the hallux using a microsurgical wrap according to the technique described by Morrison in 1978. The use of this flap needs a skin graft of the dorsal aspect of the second toe, but, as described by several authors, can produce scarring dystrophic areas and modifications of foot support.

Another surgical approach allowing valid coverage is the first dorsal metatarsal artery island flap [5] obtained from the dorsum of the foot. This flap has a pedicle long enough to provide coverage of the interphalangeal joint area, and, if needed, it can be used to cover most of the distal area of the hallux. Because of the reduced dimensions of this flap and the anatomical variations of the pedicle, a more accurate surgical technique is required in comparison to the previously described flaps. The advantage of this flap is the acceptable morbidity of the donor site.

For all of these reasons, the reconstructive solution ideally has to be found in the surrounding dorsal area of the lesion, avoiding the involvement of the teguments undergoing direct pressure, particularly the plantar aspect of the foot [11].

The long experience in our surgical unit with the use of subcutaneous flaps as well as the decisively favorable results obtained in hand surgery has led us to use this reconstructive methodology in hallux lesions. Several advantages offered by these flaps for use in this anatomical setting to cover small and medium-sized defects are to be emphasized: first, the vitality of these flaps, supplied by the perforating vessels that penetrate the flap from its base. As previously described, in the available literature dimensional standards for this flap cannot be found, but the right flap size is to be defined by the ratio between the lesion area and the proximal limit of the flap dissection. This surface, to guarantee the survival of the flap, should ensure an area to base ratio of $4: 1$. Second, subcutaneous flaps do not impose the sacrifice of important vessels and still possess very good vascularization. Their vitality is maintained even with a twisting of $180^{\circ}$. They keep a good vascularization provided that they are not compressed in the reflection area. Third, they can be used upside down, a feature that makes them much more versatile than the traditional skin-covered pedicle flaps. Finally, the scar sequelae of the donor area are minimal and particularly favorable from the esthetic point of view.

To the best of authors' knowledge, this application has not been reported in the literature so far in this anatomical site.

The technique might be worth knowing both for orthopedic and plastic surgeons, as it may represent a safe, less invasive solution for most tegumentary problems of the dorso-medial side of the first ray, also when we have to treat difficult situation like tegument infections.

Conflict of interest statement The authors declare that they have no conflict of interest related to the publication of this manuscript.

\section{References}

1. Mahan KT, Feehery RV (1991) Flexor hallucis brevis muscle flap. J Foot Surg 30(3):284-288

2. Governa M, Barisoni D (1996) Distally based dorsalis pedis island flap for a distal lateral electric burn of the big toe. Burns 22(8):52-65

3. Sakay S (1976) A distally based island first metatarsal artery flap for the coverage of a distal plantar defect. British J Plast Reconstr Surg 29:209

4. Smith A, Aarons JA, Reyes R et al (1995) Distal foot coverage with a reverse dorsalis pedis flap. Ann Plast Surg 34:191

5. Ishakawa K, Isshiki N, Susuki S et al (1987) Distally based dorsalis pedis flap for coverage of the distal portion of the foot. British J Plast Reconstr Surg 40:521

6. Hayashi A, Maruyama Y (1993) Reverse fist dorsalis metatarsal artery flap for reconstruction of the distal foot. Ann Plast Surg 31(2):112-117

7. Julien P, Marcinko D, Gordon S (1988) Reconstruction of soft tissue defects about the great toe. J Foot Surg 27(2):116-120

8. Clodius L (2002) Free grafts of gliding tissue-principles and practice. Eur J Plast Surg 25:123

9. De Lorenzi F, Vaienti L (2000) Subcutaneous tissue flaps: theoretical principles and clinical applications. Eur J Plast Surg 23:267

10. Lai CS, Lin SD, Yang CC, Chou CK (1991) Adipofacial turn over flap for the reconstruction of the dorsum of the foot. British $\mathrm{J}$ Plast Reconstr Surg 44:170

11. Cheng M-H, Ulusal BG, Wei F-C (2006) Reverse first dorsal metatarsal flap for reconstruction of traumatic defects of dorsal great toe. J TRAUMA Inj Infect Crit Care 60(5):1138-1141 